\title{
Editorial
}

\section{Toward Scalable Clinical Decision Support}

In this special issue of the Open Medical Informatics Journal, four papers are presented describing various approaches to the general problem of scaling clinical decision support (CDS) - providing CDS across potentially multiple electronic medical record systems (EMR) in varying clinical practice environments, and the methods of representing, encoding, and sharing knowledge to support such services. Each describes and employs a variety of techniques to approach critical problems in this area of clinical informatics research and development: knowledge translation and specification, knowledge aggregation and dissemination, and knowledge implementation for CDS in EMRs, and other forms of health IT. These papers provide an excellent overview of current research and development activities in this area, and hold great promise for describing methods that ultimately will enable widespread adoption and use of best practices and current clinical guideline knowledge at scale.

Current health policy in the US and other countries places great emphasis not only on the adoption of health information technology (HIT) in clinical practice, but also on its ability to achieve desired clinical outcomes through 'meaningful use' [1], or effective use of HIT at the point of care. With the unprecedented move to adopt HIT in clinical practice, a significant barrier to the effective use of HIT, and CDS [2] in particular, is now apparent - the non-availability of knowledge required for implementation in multiple, disparate EMR systems in a form that makes it easy for the healthcare organization to readily implement the knowledge. Absent the knowledge in such a form, each organization must implement CDS, replicating a nontrivial effort. Even when the end-user is sophisticated, and the EMR provides adequate tools for authoring and implementing clinical knowledge within the system, often the process of translating knowledge from a clinical practice guideline (CPG), arriving at consensus, and maintaining the knowledge in an appropriate current state, reflecting current best, or usual and customary, practices is beyond the capacity of most healthcare delivery systems.

The adoption of CDS would likely increase with more availability of knowledge in a form that can be rapidly implemented within heterogeneous organization. However, there are two major challenges in making CDS knowledge applicable across organizations. First, each organization varies in its workflows, policies, and availability of resources, thus affecting the implementation of CDS. Second, EMR systems have significant differences in function and technology. For example, differences in data structures across EMRs make it difficult to reference patient data in a commonly understood manner in the CDS knowledge artifacts.

This Special Issue focuses on the steps that take CPGs and transform them into a variety of forms for implementation through a variety of pathways into an EMR system to provide CDS at the time of clinical decision-making - the process may be described generally as knowledge engineering for CDS. It also addresses issues related to knowledge management for CDS, primarily around dissemination of the knowledge.

Let us turn now to the central, key issues in knowledge engineering for the effective application of clinical knowledge as CDS in HIT at scale, across multiple disparate EMR systems - knowledge translation and specification, knowledge aggregation and dissemination, and knowledge implementation. We discuss each in turn, and then highlight how the papers in this Special Issue address these key issues of knowledge aggregation, knowledge dissemination, and knowledge implementation.

Many investigators and EMR developers have wrestled with the problem of how to quickly and effectively translate clinical knowledge from CPGs into a form that is implementable within a EMR via one mechanism or another. Various efforts have attempted to develop knowledge artifacts from CPGs that may be implemented within EMRs - either in a proprietary representation for a single vendor's EMR, or in more portable formats that theoretically could be imported within several distinct EMRs. The paper by Hatsek et al, describes an approach to and a set of tools for gradual and progressive knowledge translation. This approach allows the most appropriate knowledge formalism to be used where necessary: for example, a human readable abstraction may be used by subject matter experts to arrive at consensus, while a more specified representation may be used by knowledge engineers who are creating a more executable form, such as a business rule.

The paper by Greenes et al, describes the Morningside Initiative, a public-private partnership in the US, that aims to foster sharing of CDS knowledge through a repository it is creating. The initiative is working on the organizational framework required for knowledge sharing. Such a framework is an essential complement to the technical work described in the other papers in this issue, if CDS is to be widely used.

The first paper by Kawamoto et al describes the use of a service-oriented architecture and standardized web-services in a novel approach to providing CDS at scale. The web-services approach may certainly facilitate broad dissemination of CDS, though only to those EMRs that have the capacity to discover, interpret, and subscribe to such a service and use the inference provided by a remote service successfully within their application framework. The key issue in scalable CDS via web-services, however, is standardization of the transactions including the underlying clinical data formats. In exchange, however, for data standardization and implementation of the ability to consume such CDS services in EMRs, this approach could allow the fastest 
path toward broad dissemination of CDS, and avoid certain localization problems that have vexed the broad adoption of CDS to date.

Another paper by Kawamoto et al describes the progress in the development of CDS-related standards. Given the heterogeneous state of EMR design and underlying architectures, and the nascent state of research in scalable CDS, it is highly likely that for some time to come a combination of methods and approaches will be necessary to achieve implementation of CDS at scale. The lack of standardization of knowledge representation, of clinical data used in CDS routines, and of EMR functional models, will continue to stymie broad implementation of CDS embedded in EMRs and those delivered through standardized CDS web services.

\section{CONCLUSION}

In this Special Issue we present papers from leading investigators and developers that describe various approaches to providing CDS at scale - across multiple, disparate EMR systems. Taken together, they describe methods that may be combined to begin to address the central issues of knowledge translation and specification, knowledge aggregation and dissemination, and knowledge implementation for CDS at scale.

\section{REFERENCES}

[1] Medicare and Medicaid Programs; Electronic Health Record Incentive Program. Washington, D.C.: Centers for Medicare \& Medicaid Services. HHS.; 2010.

[2] Osheroff J, Pifer E, Teich J, Sittig D, Jenders R. Improving Outcomes with Clinical Decision Support: An Implementer's Guide. 1st Ed. Productivity Press 2005.

Blackford Middleton

(Co-Guest Editor)

Partners HealthCare System

Harvard Medical School

Bostan, MA

USA

\author{
Aziz Boxwala \\ (Guest Editor) \\ University of California at San Diego \\ 9500 Gilman Drive \\ MS 0728 \\ La Jolla \\ CA 92093-0728 \\ USA \\ E-mail: aboxwala@ucsd.edu
}

(C) Boxwala and Middleton; Licensee Bentham Open.

This is an open access article licensed under the terms of the Creative Commons Attribution Non-Commercial License (http://creativecommons.org/licenses/by-nc /3.0/) which permits unrestricted, non-commercial use, distribution and reproduction in any medium, provided the work is properly cited. 